\title{
A community based study of failure to thrive in Israel
}

\author{
David S Wilensky, Gary Ginsberg, Michal Altman, Theodore H Tulchinsky, \\ Fiameta BenYishay, Judy Auerbach
}

\begin{abstract}
Objective-To examine the characteristics of infants suffering from failure to thrive in a community based cohort in Israel and to ascertain the effect of failure to thrive on their cognitive development.

Methods-By review of records maintained at maternal and child health clinics in Jerusalem and the town of Beit Shemesh, epidemiological data were obtained at age 15 months on a cohort of all babies born in 1991 . For each case of failure to thrive, a matched control was selected from the same maternal and child health clinic. At age 20 months, cognitive development was measured, and at 25 months a home visit was carried out to assess maternal psychiatric status by questionnaire, and the HOME assessment was performed to assess the home environment.
\end{abstract}

Results-3.9\% of infants were found to have fallen below the third centile in weight for at least three months during the first year of life. Infants with failure to thrive did not differ from the general population in terms of obstetric or neonatal complications, birth order, or parents' ethnic origin, age, or years of education. The infants with failure to thrive did have lower birthweights and marginally smaller head circumferences at birth. Developmental assessment at 20 months of age showed a DQ of $99.7 v 107.2$ in the matched controls, with $11.5 \%$ having a DQ below 80, as opposed to only $4.6 \%$ of the controls. No differences were found in maternal psychiatric problems as measured by a self report questionnaire. There were, however, significant differences in subscales of the HOME scale.

Conclusions-(1) Infants who suffered from failure to thrive had some physiological predispositions that put them at risk; (2) failure to thrive may be an early marker of families providing suboptimal developmental stimulation.

(Arch Dis Child 1996;75:145-148)

Keywords: failure to thrive, growth, mental development, outcome.

Behavioural Sciences,

Ben Gurion University, Beer Sheva

J Auerbach

Correspondence to: Dr D Wilensky, Jerusalem Child Development Centre Jaffa Rd 157, Jerusalem, Israel.

Accepted 14 May 1996
Failure to thrive (defined as growth deceleration to a point below the third centile in weight in a child born in the normal range) continues to provide a major challenge in clinical paediatric practice. A review article by Frank and Zeisel ${ }^{1}$ reported that the incidence of failure to thrive was nearly $10 \%$ in primary care settings in low income areas, with inner city emergency departments showing an even higher rate. Failure to thrive also accounts for $3-5 \%$ of all paediatric hospital admissions, ${ }^{2}$ and for almost twice as many admissions as in infants without failure to thrive $(29.8 \% v$ $15.2 \%)^{3}$

The vast majority of studies on failure to thrive have been based on series of children admitted to hospital because of failure to thrive. As Drotar has pointed out, ${ }^{4}$ selection bias and other methodological faults may have skewed the results of previous studies on failure to thrive. The picture of major psychosocial dysfunction in many of the hospital inpatients may reflect a selection bias and not necessarily characterise the profile of cases being managed in the paediatrician's office without ever requiring hospital admission.

The few studies which have used community based samples of failure to thrive support this hypothesis. Skuse et $a l^{5}$ in a prospective community based study in London, found little difference in the psychiatric state of mothers of failure to thrive infants as compared to mothers of matched controls, but instead found evidence suggestive of minor oromotor dysfunction and temperamental differences in a significant number of the infants suffering from failure to thrive. Skuse also found a significant difference in cognitive functioning on follow up of the failure to thrive infants when compared with case matched controls, a finding with major public health implications.

In this study we examine the epidemiological characteristics of a community based nonreferred sample of failure to thrive children in a different cultural setting, as well as cognitive outcome at later follow up.

\section{Methods}

Our study was carried out by a retrospective review of the records held in maternal and child health clinics of a community based cohort of all infants born in 1991 in three neighbourhoods of Jerusalem and the total population of Beit Shemesh, a town of about 25000 population. The three neighbourhoods in Jerusalem reflected upper, mixed, and lower socioeconomic levels. In Beit Shemesh, $99 \%$ of the population attended the maternal and child health clinics for well baby care, and in Jerusalem $90-95 \%$ attended. All records were reviewed after the infants had reached 15 months of age. Height, weight, and head circumfer- 
Table 1 Characteristics of failere to thrive (FTT) compared with non-FTT children (from review of records). Values are means (SD)

\begin{tabular}{lll}
\hline & $F T T(n=55)$ & Non-FTT $(n=1352)$ \\
\hline Birth weight (g) & $3074.2(385.5)$ & $3229.9(504.1)^{\star}$ \\
Head circumference at birth (cm) $^{\star}$ & $33.75(1.12)(\mathrm{a})$ & $34.16(1.46)$ \\
Normal pregnancy $^{1}$ & $80.0 \%$ & $82.5 \%$ \\
Normal delivery $^{2}$ & $74.5 \%$ & $77.0 \%$ \\
No neonatal complications $^{3}$ & $87.3 \%$ & $91.3 \%$ \\
Gestational age (weeks) $_{\text {Parity }}^{39.8(1.2)}$ & $39.6(2.4)$ \\
Maternal age & $2.91(1.58)$ & $2.66(1.65)$ \\
Maternal education (years) & $30.51(5.33)$ & $29.49(5.60)$ \\
Gender of child (\% males) & $13.84(2.87)$ & $13.43(2.81)$ \\
Recorded medical problems & $56.4 \%$ & $51.7 \%$ \\
Hypotonia in first year of life & $0.84(1.03)$ & $0.52(0.83)$ \\
\hline
\end{tabular}

(a) $\mathrm{n}=44$.

${ }^{1}$ Complications defined as gestational diabetes, bleeding, viral infection or medications in the first trimester, toxaemia, smoking.

${ }^{2}$ Complications defined as breech, vacuum, or forceps delivery, caesarean section, fetal distress, pathological placenta.

${ }^{3}$ Complications defined as Apgar $<7$, respiratory distress, neonatal seizures, bilirubin $>20$ or exchange transfusion, congenital malformations.

$\star \mathrm{p}<0.05$. scale reflecting the examiner's subjective assessment of the infant's behavioural repertoire during the examination, was also filled out by the examiner at the end of the session. Follow up anthropometric data were obtained at this visit. At 25 months the psychologist made a home visit, at which time data were obtained from the mother on the infant's feeding and medical history, mother's psychiatric status (the brief symptom inventory), ${ }^{7}$ and mother's perceptions of the infant's temperament (Bates infant characteristics questionnaire), ${ }^{8}$ and an assessment of the home environment (HOME questionnaire) ${ }^{9}$ was recorded.

Statistical analysis was carried out using SAS statistical software to compute $\chi^{2}$ tests, $t$ tests, and matched pairs $t$ tests on the variables. Logistic regression analysis with failure to thrive as the dependent variable was carried out, and a multiple regression analysis with MDI as the dependent variable was undertaken in order to assess potential covariates. Values are given as mean (SD).

visit, were recorded, as was demographic background, obstetric and neonatal history, results of paediatric examinations, and any references to medical problems reported in the nurses' notes. Babies were measured an average of four times in the first sixth months of life, twice in the second six months, and again at 15 months. The total cohort numbered 1452 births.

Failure to thrive was defined as falling below the 3 rd centile in weight (using NCHS growth curves $^{6}$ ) for a duration of at least three months. This definition has the advantage of providing data comparable with other published studies, but it misses other infants who may have been nutritionally compromised and fallen across two or more weight centiles without falling absolutely below the third centile. All infants born under $2500 \mathrm{~g}$ or before 37 weeks' gestational age were excluded, as were infants whose weight to height ratio was above $10 \%$. Fifty five cases were identified under these criteria. Subsequently, a matched control from the same maternal and child health clinic, born in the same month as the failure to thrive case, was chosen. Controls were selected by taking the baby with the nearest birth date who met the additional matching criteria of maternal education, ethnic origin (based on maternal grandmother), maternal age, parity, and infants' birth weight. Of the $\mathbf{5 5}$ failure to thrive cases, one refused to participate in the study, one had moved out of the area, and three cases with significant medical causes for their failure to thrive (coeliac disease, recurrent urinary tract infections, and tracheostomy) were excluded to avoid confounding medical variables in the outcome study. None of the selected controls refused participation.

A cross sectional study was carried out on the remaining 50 matched pairs. A developmental psychologist who was blind to the infants' nutritional status performed the Bayley developmental assessment on the failure to thrive and control infants at an average age of 20 months (SD 3 ). The Bayley test generates a score reflecting mental development (the MDI) and one reflecting motor development (PDI). The Bayley infant behaviour record, a

\section{Results}

The overall incidence of failure to thrive was found to be $3.9 \%$ (3.7\% in Jerusalem and $4.5 \%$ in Beit Shemesh) among the cohort of 1407 children (after excluding small for gestational age infants, preterm infants, and transient failure to thrive which occurred in $1.5 \%$ ). Average age of onset was 8.4 (0.4) months, with only 11 cases falling below the third centile before 6 months of age. Failure to thrive infants differed from the general population in having lower initial birth weights (3074 $v 3230$ g; p < 0.05), marginally significantly smaller head circumference at birth $(33.75 v 34.16$ $\mathrm{cm} ; \mathrm{p}=0.064$ ), and marginally more recorded medical problems during the first year of life ( 0.84 diagnoses $v 0.52 ; \mathrm{p}=0.052$ ). A higher percentage of failure to thrive infants was diagnosed as being hypotonic during the first year of life $\left(7.27 \% \vee 2.27 \% ; \chi^{2}[1]=5.10\right.$; $\mathrm{p}<0.05$ ), based on the paediatric examinations recorded at the maternal and child health centres.

Logistic regression analysis was carried out with the variables birth weight, head circumference at birth, parity, maternal age, maternal years of education, paternal age, and paternal years of education. Only paternal age was found to be significantly positively associated with failure to thrive $(p<0.01)$.

Demographic data showed no differences between the groups in maternal age, birth order, or maternal years of education. There were also no significant differences in the incidence of obstetric or neonatal complications (table 1). There were also no differences related to maternal country of birth, with $68 \%$ Israeli-born, $25 \%$ born in Europe or America, $5 \%$ in North Africa, and $2 \%$ born in Asia.

At 20 months of age three fifths of the failure to thrive infants were still below the 3 rd centile for weight. The matched case-control data showed significantly lower mental development in the failure to thrive infants on the 20 month Bayley mental test score (MDI $99.7 v$ 107.2; $p<0.05$ using paired comparison test). 
Table 2 HOME results of failure to thrive (FTT) compared with matched non-FTT children (at 25 months). Values are mean (SD)

\begin{tabular}{lll}
\hline & $F T T(n=50)$ & Non-FTT $(n=50)$ \\
\hline Active stimulation & $0.84(0.17)^{1}$ & $0.92(0.11)^{\star}$ \\
Family participation in encouraging development & $0.55(0.31)$ & $0.71(0.24)^{\star}$ \\
Verbal and emotional reactivity & $0.85(0.20)$ & $0.93(0.14)^{\star}$ \\
Encouragement of maturity & $0.76(0.33)$ & $0.85(0.26)$ \\
Emotional climate & $0.77(0.11)$ & $0.78(0.11)$ \\
Growth nurturing & $0.49(0.27)$ & $0.57(0.25)$ \\
Physical environment & $0.88(0.26)$ & $0.86(0.26)$ \\
Parental involvement & $0.52(0.21)$ & $0.51(0.22)$ \\
General overall average & $0.72(0.13)$ & $0.77(0.16)$ \\
\hline
\end{tabular}

${ }^{\star} \mathrm{p}<0.05$.

${ }^{1}$ In each area the score reflects a range of optimality from $0-1$, with 1 representing the optimal score; $\mathrm{HOME}=\mathrm{HOME}$ questionnaire assessing home environment.

Table 3 Feeding difficulties of failure to thrive (FTT) compared with matched non-FTT children (at 25 months)

\begin{tabular}{lllr}
\hline & $F T T(n=50)(\%)$ & Non-FTT $(n=50)(\%)$ & \multicolumn{1}{l}{$\chi^{2}$} \\
\hline Showing hunger & 53.4 & 96.2 & $24.00 \ddagger$ \\
Closes mouth and turns head & 69.7 & 20.7 & $23.31 \ddagger$ \\
Shows pleasure at mealtimes & 76.7 & 94.3 & $6.27 \dagger$ \\
Nervous at mealtimes & 23.2 & 5.6 & $6.27 \dagger$ \\
Eats variety of foods & 48.8 & 69.8 & $4.36^{\star}$ \\
Spits out food & 65.1 & 33.9 & $9.23 \dagger$
\end{tabular}

${ }^{\star} \mathrm{p}<0.05 ; \mathrm{tp}<0.01 ; \neq \mathrm{p}<0.001$.

More failure to thrive infants than controls scored a mental development quotient below $80(11.5 \% v 4.6 \%)$. Multiple regression analysis on the total sample found mothers' education $(p<0.01)$, the HOME score, and failure to thrive to be positively associated with the Bayley mental score at 20 months. When analysing the failure to thrive group alone, maternal education and the HOME active stimulation subscale were found to be positively associated with the MDI score. Paternal education level, head circumference at birth, age of failure to thrive onset, and weight at 20 months were not predictive of mental outcome. In analysing the control group alone, years of maternal education was no longer found to be significant and only the HOME score covaried with mental development. Behavioural observations during the 20 month examination found the failure to thrive infants somewhat less sociable $(p<0.05)$ and more fearful of the examiner $(p<0.05)$.

The home visit at 25 months revealed no significant differences in current maternal psychiatric status. The HOME questionnaire, however, showed failure to thrive homes to be significantly lower $(p<0.05)$ in the subscales relating to 'verbal and emotional reactivity', 'active stimulation', and 'family participation in encouraging development' (table 2). Thus the families of failure to thrive children were less stimulating in their parenting styles.

The feeding questionnaire indicated that similar proportions of infants had been breast fed in the two groups $(88.4 \% v 86.8 \%)$, but that the failure to thrive children had been breast fed for a longer duration (15.3 $v 11.4$ weeks; NS).The average length of each breast feed, however, was significantly longer in the failure to thrive group (19.5 v $12.9 \mathrm{~min}$; $\mathrm{p}<0.05)$. The failure to thrive infants also differed significantly in their feeding histories. They had tended to fall asleep while breast feeding more often, had slept through scheduled feeding times, had refused solids more often, and still showed more difficulties related to feeding at 25 months of age (table 3). The reliability of these data are of course limited by the fact that they are based on maternal report.

Based on maternal report, failure to thrive infants were twice as likely to have been admitted to hospital in the first year of life than the control infants $\left(33.9 \% v 16.9 \% ; \chi^{2}[1]=4.03\right.$; $\mathrm{p}<0.05)$. No difference was found for the incidence of recurrent ear infections $(28 \% v$ $26 \%$ ).

The Bates questionnaire revealed no significant differences in temperament in infancy between the failure to thrive group and the matched control group.

\section{Discussion}

Our results confirm the hypothesis that a nonreferred community based sample of failure to thrive infants may have different characteristics from those found in hospital inpatients. Data were limited to questionnaire replies by the mothers, which were not concurrent with the onset of the failure to thrive, but within these limitations no difference was found in the psychiatric status of the mothers of failure to thrive infants and controls.

The data support the findings of Skuse et al, ${ }^{4}$ that failure to thrive infants may differ from birth in their behavioural characteristics and their neurological status-they were reported to be more passive, and more likely to sleep through meals, and a higher percentage was diagnosed as being hypotonic. Although we did not examine oromotor function directly, the failure to thrive infants did take longer to finish each breast feed. Thus there may be some indirect evidence for the presence of mild oromotor difficulties in the failure to thrive infants, as reported by Skuse et al. On an interactional level there was also evidence that failure to thrive infants received less appropriate developmental stimulation in their home setting, over and above the problems relating to the feeding situation. The lower scores on the HOME questionnaire in this study reconfirm the findings of previous studies. ${ }^{411}$ Despite the fact that the mothers of the failure to thrive infants did not differ significantly in terms of demographic characteristics or psychiatric status, the HOME data nonetheless suggest that their interactions with their infants were less optimal than for the matched controls.

While no attempt was made to characterise family dynamics, it is of interest that the logistic regression analysis showed that the incidence of failure to thrive covaried with increasing paternal age. One could speculate that older fathers were less available to support their wives or participate in the infant's care, thus making the child more vulnerable to suffering from failure to thrive. Relative maternal isolation and lack of social supports has been postulated as a cause for failure to thrive in other studies. ${ }^{12}$

It is hard do draw clear conclusions from retrospective data as to the aetiological factors leading to failure to thrive in our sample, but 
the data seem to be compatible with the findings of Altemeier $e t$ al, ${ }^{13}$ in their prospective study of the antecedents of non-organic failure to thrive. They reported a combination of biological vulnerability (mild perinatal problems) in combination with environmental difficulties (poor nurturing of the mothers in their own childhood, together with poor current marital relationships). In our sample, lower birth weights and smaller head circumferences may reflect biological vulnerability. Our HOME data suggest that environmental issues may also interact with initial vulnerability to create the feeding difficulties which were reported in the failure to thrive infants.

The most significant finding in public health terms is the cognitive delay in the failure to thrive infants at 20 months of age. Twice as many failure to thrive infants showed mental developmental quotients less than 80 at this age. Behaviourally, the infants also showed less sociability. The multiple regression analysis seems to support the importance of environmental factors (maternal education and HOME subscale as significant covariants) in affecting developmental outcome. The difference between failure to thrive infants and controls is interesting in this regard. It would appear that 'good enough' mothering was indeed good enough in the control group to prevent years of maternal education from having an impact on developmental outcome, whereas in the more vulnerable failure to thrive infants, years of maternal education did covary with later mental scores. Similar additive effects of biological and environmental risk factors have been noted in other at risk populations, for example, small for gestational age infants ${ }^{14}$ and preterm infants. ${ }^{15}$ Unlike Skuse, we did not find that the age of onset of failure to thrive correlated with mental development; thus in our sample there was no evidence for the effect of earlier onset of malnutrition as a major factor in later outcome.

Longitudinal studies of selected samples of failure to thrive infants have shown significant long term morbidity in cognitive and behavioural outcomes. ${ }^{16-20}$ Studies on community based samples are few and contradictory in their results. Mitchell et al found no decrease in IQ at age 4 in a failure to thrive cohort from their rural paediatric practice. ${ }^{21}$ Skuse et al, on the other hand, found significant lowering of cognitive function a failure to thrive sample from an inner city London population. If our results prove to be robust and continue to be present or even worsen on longer term follow up, then they have major implications for the need for early intervention, even for cases that do not require hospital admission, in order to try to minimise the developmental gaps which we have shown to be already present at 20 months of age. Both laboratory data ${ }^{22}$ and clinical studies ${ }^{23}$ provide evidence for the possibility of ameliorating the sequelae of failure to thrive by early intervention.

Our results show that failure to thrive, even in the context of ambulatory paediatrics, must be taken seriously as a risk factor for later cognitive development. Failure to thrive should be regarded as an early warning sign for infants biologically at risk, with family environments which may not be equipped to cope optimally with such infants and are unable to provide appropriate cognitive stimulation on top of the manifest problems with feeding.

We would like to express appreciation to Dr David Skuse for inspiring this study and for his guidance, to Rami BarGiora for his ongoing support, to Prof Asher Ornoy for his support and advice, to Sheila Wilensky, Gili Sokolovsky, and SooYan Kim for their dedication and perseverance in gathering the data, to Dr Aryeh Reissen and all the nurses from the Jerusalem Public Dr Aryeh Reissen and all the nurses from the Jerusalem Public Health System for their cooperation
all the nurses from Beit Shemesh.

1 Frank DA, Zeisel SH. Failure to thrive. Pediatr Clin North Am 1988:35:1187-206.

2 Berwick DM. Nonorganic failure to thrive Pediatr Rev 1980;1:265-80.

3 Skuse D, Pickles A, Wolke D, Reilly S. Postnatal growth and mental development: evidence for a 'sensitive period'. $f$ Child Psychol Psychiatry 1994;35:521-45.

4 Drotar D. Failure to thrive. In: Routh DK, ed. Handbook of pediatric psychology. New York: Guilford, 1988:71-107.

5 Mason JB, Mitchell JT. Nutritional surveillance. Bull WHO 1983;61:745-55.

6 Skuse DH, Wolke D, Reilly S. Failure to thrive. Clinical and developmental aspects. In: Remschmidt $\mathrm{H}, \mathrm{Scmidt} \mathrm{M}$, eds. Developmental psychopathology, child, and youth psychiatry. European perspectives. Vol 2. Gottingen: Hogrefe and Huropean perspectives.

7 Derogatis LR, Meliaratos, $N$. The brief symptom inventory:an introductory report. Psychol Med 1983;13:595-605

ry:an introductory report. Psychol Med 1983;13:595-605
8 Bates JE, Freeland CAB, Lounsbury ML. Measurement of Bates JE, Freeland CAB, Lounsbury ML. Measur
infant difficultness. Child Dev 1979;50:794-803.

9 Caldwell B, Bradley R. Home observation for the measurement of the environment. Little Rock: University of Arkansas, 1979 .

10 Rosen DW, Loale LS, Jura MB. Differentiation of organic and nonorganic failure to thrive in infancy. Pediatrics 1980 66:66-75.

11 Casey P, Bradley RH, Wortham B. Social and nonsocial home environments of children with non-organic failure to thrive. Pediatrics 1984;73:348-53.

12 Kotelchuck $M$, Newberger,EH. Failure to thrive: a controlled study of familial characteristics. $₹$ Am Acad Child Psychiatry $1983 ; 22: 322-8$.

13 Altemeier WA, O'Connor SM, Sherrod KB, Vietze PM. Prospective study of antecedents of nonorganic failure to thrive. F Pediatr 1985;106:360-5.

14 Teberg AJ, Walther FJ, Pena IC. Mortality, morbidity, and outcome of the small-for-gestational age infant. Sem Perinatol 1988;12:84-94.

15 Ella S, Greenbaum CW, Wilensky D, Ornoy A. Cognitive outcomes of Israeli schoolchildren born prematurely at very low birth weight. In: Greenbaum CW, Auerbach JG eds. Longitudinal studies of children at psychological risk:cross national perspectives. Jerusalem: Ablex Publishing Corporation, 1992:79-98.

16 Elmer E, Gregg GS, Ellison P. Late results of the 'failure to thrive' syndrome. Clin Pediatr 1969;8:584-9.

17 Chase H, Martin H. Undernutrition and child development. $N$ Engl f Med 1979;282:933-9.

18 Oates RK, Peacock A, Forrest D. Long-term effects of nonorganic failure to thrive. Pediatrics 1985;75:36-40.

19 Glaser HH, Heagerty MC, Bullard DM, Pivchick EC Physical and psychological development of children with early failure to thrive. $\mathcal{F}$ Pediatr 1968;73:690-8.

20 Ornoy A,Suan G, Tannenbaum A. Effects of NFTT on long term development of children. In: Ornoy A, ed. Proceeding of the National Symposia of the Child Develpment Centers in Israel 1989/1990. Jerusalem: Almog, 1991:157.

21 Mitchell WG, Gorell RW, Greenberg RA. Failure to thrive: a study in a primary care setting. Pediatrics 1980;65:971-7.

22 Davies CA undernutrition and subsequent differential of early-life undernutrition and subsequent differential environments on the dendritic branching of pyramidal
cortex. $\mathcal{F}$ Comp Neurol 1983;218:345-50.

23 Hicks LE, Langham RA, Takenaka J. Cognitive and health measures following early nutritional supplementation: a measures following early nutritional supplement
sibling study. Am $\mathcal{f}$ Public Health 1982;72:1110-8. 